\title{
Article \\ Factors Related to Health Risk Communication Outcomes among Migrant Workers in Thailand during COVID-19: A Case Study of Three Provinces
}

\author{
Ratchadaporn Papwijitsil ${ }^{1, *(\mathbb{D})}$, Hathairat Kosiyaporn ${ }^{2}$, Pigunkaew Sinam ${ }^{2}$, Mathudara Phaiyarom ${ }^{2} \mathbb{1}$, \\ Sataporn Julchoo ${ }^{2}$ and Rapeepong Suphanchaimat ${ }^{1,2}$ \\ 1 Field Epidemiology Training Program (FETP), Division of Epidemiology, Department of Disease Control, \\ Ministry of Public Health, Nonthaburi 11000, Thailand; rapeepong@ihpp.thaigov.net \\ 2 International Health Policy Program (IHPP), Ministry of Public Health, Nonthaburi 11000, Thailand; \\ hathairat@ihpp.thaigov.net (H.K.); pigunkaew@ihpp.thaigov.net (P.S.); mathudara@ihpp.thaigov.net (M.P.); \\ sataporn@ihpp.thaigov.net (S.J.) \\ * Correspondence: kaenoipap@gmail.com
}

\section{check for} updates

Citation: Papwijitsil, R.; Kosiyaporn, H.; Sinam, P.; Phaiyarom, M.; Julchoo, S.; Suphanchaimat, R. Factors Related to Health Risk Communication Outcomes among Migrant Workers in Thailand during COVID-19: A Case Study of Three Provinces. Int. J. Environ. Res. Public Health 2021, 18, 11474. https://doi.org/10.3390/ ijerph182111474

Academic Editor: Paul B. Tchounwou

Received: 14 August 2021

Accepted: 29 October 2021

Published: 31 October 2021

Publisher's Note: MDPI stays neutral with regard to jurisdictional claims in published maps and institutional affiliations.

Copyright: (c) 2021 by the authors. Licensee MDPI, Basel, Switzerland. This article is an open access article distributed under the terms and conditions of the Creative Commons Attribution (CC BY) license (https:/ / creativecommons.org/licenses/by/ $4.0 /)$.

\begin{abstract}
Coronavirus disease 2019 (COVID-19) is a newly emerging infectious disease, and risk communication is one of several public health emergency responses. During the pandemic, many migrant workers in Thailand experienced barriers that hamper access to health information. This study aims to explore factors related to the outcomes of health risk communication, including awareness of public health measures and preventive practices. We conducted a cross-sectional survey on migrants between January and April 2021 using cluster sampling in Phuket, Ranong, and Samut Sakhon. In the descriptive analysis, we presented the median, proportion, and ratio, while in the inferential analysis, we employed a logistic regression with robust standard errors. Although a total of 303 participants were initially included in this study, the final number was narrowed down to 288 samples due to insufficient information required for the analysis. Frequent reception of health information and primary school education showed a statistically significant association with preventive practices. Middle-aged migrant workers demonstrated a significantly lower level of preventive practices than younger migrant workers. A longer stay in Thailand was significantly related to a lower degree of awareness toward public health measures. Thus, it is necessary to promote the accessibility of health information among migrant workers in Thailand, especially those who have lived in Thailand for more than eight years, are older, and have no formal education.
\end{abstract}

Keywords: risk communication; migrants; practice; awareness; COVID-19; Thailand

\section{Introduction}

The coronavirus disease 2019 (COVID-19) was first recognized in late 2019 [1-3] and was later declared by the World Health Organization (WHO) on 30 January 2020 to be a Public Health Emergency of International Concern (PHEIC) [4]. In response, the WHO aimed to launch a global coordinated effort for effective preparedness and response to COVID-19. According to a WHO report on 20 October 2021, the COVID-19 pandemic has affected over 241 million people and caused 4.9 million deaths in more than 200 countries [5].

Thailand was the first country outside China to report the presence of COVID-19. The first wave of COVID-19 in Thailand started with clusters of infections related to imported cases from other countries and local transmission [6]. In response to this, the Thai Government introduced several non-pharmaceutical interventions to curb the outbreak, for instance, international travel bans and social distancing [7]. Risk communication was included in the Incident Command System (ICS) as an essential component for health emergency preparedness and response [8]. The Thai Government established the Center for 
COVID-19 Situation Administration (CCSA) as the governing body of the ICS [9]. Due to these combined measures and a resilient health system, Thailand succeeded in containing the first wave of COVID-19 in 2020 [10,11].

However, from late December 2020, Thailand faced a new wave that was more severe than the previous one [12]. This second wave was believed to have originated from migrant workers in the inner city of Samut Sakhon [13], a province within the vicinity of Bangkok and a major residential area hosting a large number of migrants. Thailand is known as one of the popular cross-broader destinations in Southeast Asia, and the number of migrants in Thailand was approximately 4.9 million in 2018; most of the migrants relocated from neighboring countries, including Cambodia, Lao PDR, Myanmar, and Viet Nam (CLMV nations) [14], with the greatest number of migrants coming from Myanmar [15].

The vulnerability of migrants has been highlighted during the COVID-19 pandemic. Most migrants live in crowded housing with poor hygiene and have limited capacity for social distancing, leading to the spread of infection [16]. Migrants may also experience difficulties in accessing health information and services due to financial hardship and language barriers [17].

Prior studies in Thailand have found that migrant health workers (MHWs), migrant health volunteers (MHVs), and village health volunteers (VHVs) were utilized in the Government's initiative to provide access to health information to migrants, especially for pandemic preparedness in previous emerging diseases such as influenza (H1N1) [18,19]. They also found that while most migrants had a high level of knowledge about the disease, they did not agree with preventive measures such as border control or social distancing. Moreover, education and reception of information from MHW and television were significantly associated with higher knowledge about health issues. During COVID-19, the International Organization for Migration conducted rapid studies in 2020 and found that approximately one-third of migrants did not understand health information [20-22]. Studies that explore factors relating to behavioral outcomes and health risk communication specifically among migrants in Thailand during COVID-19 are also lacking.

To address this gap in knowledge, it is necessary to investigate factors influencing the reception of health information. The health belief model (perceived risk, perceived severity, perceived benefits, and perceived barriers) was used as an overarching concept for this study [23]. Therefore, this study aims to explore the outcomes of health risk communication, including the level of awareness and self-reported COVID-19 prevention among migrants, along with other associated factors. It is hoped that the findings from this study will provide impactful contributions toward public health research on migrant health in Thailand and result in more comprehensive migrant health policies.

\section{Materials and Methods}

\subsection{Study Design and Setting}

A cross-sectional survey was conducted between January and April 2021 on migrant workers aged 15 years and above in the three provinces of Phuket, Ranong, and Samut Sakhon. These provinces were chosen since they have some of the most densely migrantpopulated areas in Thailand. Surveys were distributed to survey participants in the main district of each province.

\subsection{Sample Size and Sampling Method}

Cluster sampling was used as the basis for obtaining our samples. We purposively selected two main migrant communities in each province that were identified by local providers or representatives of non-government organizations (NGOs) who were familiar with the field. Then, we randomly selected households based on the proportion of households in the community compared with the total number of households in the study. We randomly selected a migrant from each household (likely to be either the household head or the household head's spouse). The sample size calculation was based on the following formula, $\mathrm{n}=\operatorname{deff} \times\left(\mathrm{Z}^{2} P(1-P) / \mathrm{e}^{2}\right)$ where deff $($ design effect $)=2 ; \mathrm{Z}$ for $5 \%$ type-1 error $=1.96$; 
$P=0.96$ (proportion of people with regular hand washing during COVID-19) [24], e = 0.05. According to this formula, the calculated sample size was 242 but we expanded the sample size to 303 to account for a $20 \%$ non-response rate or incomplete responses.

\subsection{Data Collection}

A bilingual (Thai and Myanmar) interviewer-assisted questionnaire was used in this study. We asked MHWs and MHVs in the field to help with the interviews. The interviewers described the purpose, risks, and benefits of the study and obtained informed consent from participants before collecting data. The questionnaire was mainly paperbased, but online questionnaires were also provided to participants for their convenience.

\subsection{Questionnaire Design}

The questionnaire consisted of three sections: (i) personal information; (ii) awareness toward public health measures on COVID-19 and self-reported practices during COVID19; (iii) questions about health literacy levels, modes, and frequency to obtain health information, the health belief model, and other potential confounders such as knowledge about disease and attitude toward public health measures.

Personal information consisted of questions about gender, age, ethnicity, family status, occupation, education, income, length of stay in Thailand, medical insurance, Thai language fluency, and resources supporting access to information and practices.

Questions about knowledge and self-reported practices for COVID-19 were adapted from the Department of Disease Control (DDC), Thailand's website [25], while attitude and awareness of public health measures on COVID-19 were adapted from CCSA's announcement [26]. Health literacy questions were adapted from Osborne et al. [27] and questions for assessing constructs of the health belief model were modified from the version found in Jones et al. [23].

The questionnaire was pilot tested and underwent content validation by five experts. Then, we calculated the index of item objective congruence (IOC) and revised the questionnaire until each question had an IOC score of more than 0.5. We conducted a 30-participant pilot survey in Phuket to test the questionnaire's reliability and clarity. We calculated Cronbach's alpha coefficient for each set of questions based on the theme and found that all of them exceeded 0.7, reflecting satisfactory reliability. An instructional manipulation check (IMC) question was placed in the middle of the questionnaire to ensure that the participants had adequately read the instructions for all questions.

\subsection{Data Analysis}

For descriptive analysis, we used median and percentiles for continuous data, and proportions and ratios were used for categorical data. In the inferential analysis, we used logistic regression with robust standard error for both univariate and multivariate analyses. STATA $^{\circledR}$ version 14 was used for the calculation. In the multivariate analysis, we selected only variables that had a $p$-value $\leq 0.1$ from the univariate analysis into the model and presented the results in the form of an adjusted odds ratio (AOR) with a 95\% confidence interval (CI).

The analytic part consisted of two main strands: (i) awareness of public health measures and (ii) self-reported preventive practices during COVID-19. For the first strand, demographic data and sources of information were designated as independent variables, whereas awareness of public health measures on COVID-19 served as a dependent variable. For the latter strand, the independent variables covered demographic data, resources for supporting preventive practices, the overall frequency of receiving health information from all sources, health literacy, knowledge about the disease, awareness, and attitudes toward public health measures, and the four constructs of the health belief model (perceived risk, perceived benefit, severity of illness, and perceived barriers), while self-reported preventive practices toward COVID-19 was considered a dependent variable (Table 1). The arrangement of each variable is presented in Table 2. 
Table 1. Dependent variables and independent variables for analytic study.

\begin{tabular}{|c|c|}
\hline Dependent Variables & Independent Variables \\
\hline $\begin{array}{l}\text { Awareness of public health measures } \\
\text { on COVID-19 }\end{array}$ & $\begin{array}{ll}\text { - } & \text { Demographic data } \\
- & \text { Source of information } \\
0 & \text { From health professionals } \\
0 & \text { From community members } \\
0 & \text { From mass media } \\
& \text { From social media }\end{array}$ \\
\hline $\begin{array}{l}\text { Self-reported preventive practices } \\
\text { during COVID-19 }\end{array}$ & $\begin{array}{l}\text { Demographic data } \\
\text { Resources for supporting preventive practices } \\
\text { Health literacy } \\
\text { Overall frequency of receiving health information from } \\
\text { all sources } \\
\text { Knowledge about disease } \\
\text { Awareness and attitudes toward public health measures } \\
\text { The four constructs of the health belief model (perceived } \\
\text { risk, perceived benefit, severity of illness, and } \\
\text { perceived barriers) }\end{array}$ \\
\hline
\end{tabular}

Table 2. Characteristics of the variables and variable management.

\begin{tabular}{|c|c|c|c|}
\hline Theme & Variables & Type of Variable & Classification \\
\hline \multirow{9}{*}{ Demographic data } & Gender & Categorical & $\begin{array}{ll}- & \text { Male } \\
\text { - } & \text { Female }\end{array}$ \\
\hline & Age (years) & $\begin{array}{l}\text { Continuous, then changed } \\
\text { to Categorical }\end{array}$ & $\begin{array}{ll}\text { - } & \leq 24 \\
\text { - } & 25-59 \\
\text { - } & \geq 60\end{array}$ \\
\hline & Ethnicity & Categorical & $\begin{array}{ll}\text { - } & \text { Myanmar } \\
\text { - Non-Myanmar (Karen, Mon, } \\
\text { Dawei, etc.) }\end{array}$ \\
\hline & Length of stay in Thailand (years) & $\begin{array}{l}\text { Continuous, then changed } \\
\text { to categorical (cut point } \\
\text { by median) }\end{array}$ & $\begin{array}{ll}\bullet & \leq 8 \\
\bullet & >8\end{array}$ \\
\hline & Education & Categorical & $\begin{array}{l}\text { - } \quad \text { No formal education } \\
\text { - } \quad \text { Primary school } \\
\text { - } \quad \text { Secondary school and above }\end{array}$ \\
\hline & Occupation & Categorical & $\begin{array}{ll}\text { - } & \text { Unemployed } \\
\text { - } & \text { Factory worker } \\
\text { - } & \text { Agriculture/fishery } \\
\text { - } & \text { Construction worker } \\
& \text { Business/housemaid/ } \\
& \text { waiter/other }\end{array}$ \\
\hline & $\begin{array}{l}\text { Family members (aged } 15 \text { years or } \\
\text { above) }\end{array}$ & $\begin{array}{l}\text { Continuous then changed to } \\
\text { categorical (using the } \\
\text { median as the cut-off point) }\end{array}$ & $\begin{array}{l}\quad \quad<2 \text { people } \\
-\quad \geq 2 \text { people }\end{array}$ \\
\hline & $\begin{array}{l}\text { Income (minimum wage: Phuket } \\
\text { 336, Ranong 315, Samut Sakhon } \\
331 \text { Baht/day }{ }^{\beta} \text { ) }\end{array}$ & $\begin{array}{l}\text { Categorical then changed } \\
\text { to categorical }\end{array}$ & $\begin{array}{ll}\text { - } & \text { Lower than minimum wage } \\
\text { - } & \text { Equal to minimum wage } \\
\text { Higher than minimum wage }\end{array}$ \\
\hline & $\begin{array}{l}\text { Thai Reading Comprehension } \\
\text { Thai Listening Comprehension }\end{array}$ & Categorical & $\begin{array}{ll}\text { - } & \text { Fully understand } \\
\text { - } & \text { Partially understand } \\
\text { - } & \text { Cannot understand }\end{array}$ \\
\hline
\end{tabular}


Table 2. Cont.

\begin{tabular}{|c|c|c|c|}
\hline Theme & Variables & Type of Variable & Classification \\
\hline & Health insurance & Categorical & $\begin{array}{l}\text { - } \quad \text { Social Security Scheme }{ }^{\mathrm{a}} \\
\text { - } \quad \text { Health Insurance Card Scheme }{ }^{\mathrm{b}} \\
\text { - }\end{array}$ \\
\hline \multirow{3}{*}{ Access to resources } & $\begin{array}{ll}\text { - } & \text { Electricity } \\
\text { - } & \text { Phone signal } \\
\text { - } & \text { Internet signal } \\
\text { - } & \text { Face mask } \\
\text { - } & \text { Soap } \\
\text { - } & \text { Alcohol gel } \\
\text { - } & \text { Tap water }\end{array}$ & Categorical & $\begin{array}{ll}\text { - } & \text { Good access } \\
\text { - } & \text { Partial access } \\
\text { - } & \text { Cannot access }\end{array}$ \\
\hline & $\begin{array}{l}\text { Resources supporting access to } \\
\text { information } \\
\text { (Total score for access to electricity, } \\
\text { phone signal, and internet signal: } \\
\text { 1-3 for each) }\end{array}$ & $\begin{array}{l}\text { Categorical (using the } \\
\text { median as the } \\
\text { cut-off point) }\end{array}$ & $\begin{array}{ll}- & \text { Low access } \\
- & \text { High access }\end{array}$ \\
\hline & $\begin{array}{l}\text { Resources supporting for } \\
\text { preventive practices } \\
\text { (Total score for access to face mask, } \\
\text { soap, alcohol gel, and tap water: } \\
1-3 \text { for each) }\end{array}$ & $\begin{array}{l}\text { Categorical (using the } \\
\text { median as the } \\
\text { cut-off point) }\end{array}$ & $\begin{array}{ll}- & \text { Low access } \\
\text { - } & \text { High access }\end{array}$ \\
\hline Health literacy & $\begin{array}{l}\text { Health literacy } \\
\text { (Total score for health literacy } \\
\text { questions: } 1-3 \text { for each question, } 12 \\
\text { questions in total) } \\
\text { (Example: I can find information on } \\
\text { health problems that concern me. } \\
\text { (Agree/Neutral/Disagree)) }\end{array}$ & $\begin{array}{l}\text { Categorical (cut-off point } \\
\text { of } 60 \% \text { of total score } \gamma \text { ) }\end{array}$ & $\begin{array}{l}\text { - } \quad \text { Low health literacy } \\
\text { - } \quad \text { High health literacy }\end{array}$ \\
\hline \multirow[b]{2}{*}{$\begin{array}{l}\text { Source of } \\
\text { information }\end{array}$} & $\begin{array}{ll}\text { Sources } \\
\text { - } & \text { Health professional } \\
\text { (Thai staff) } \\
\text { - } \quad \text { MHW/MHV/VHV } \\
\text { - } \quad \text { Family/friends } \\
\text { - } \quad \text { Community/religious leader } \\
\text { - } \quad \text { Boss/employer } \\
\text { - } \quad \text { Television/radio } \\
\text { - } \quad \text { Newspaper/posters/leaflets } \\
\text { - } \quad \text { Social media (Facebook, } \\
\quad \text { YouTube, etc.) }\end{array}$ & Categorical & $\begin{array}{ll}- & \text { Did not receive (score 1) } \\
- & \text { Sometimes (score 2) } \\
\text { - } & \text { Frequently (score 3) }\end{array}$ \\
\hline & 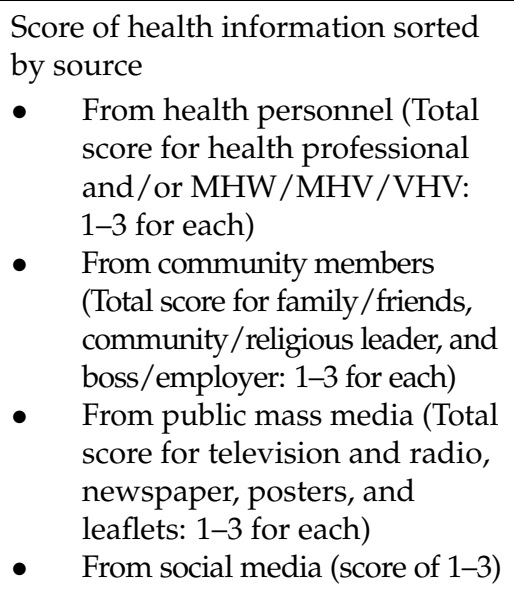 & $\begin{array}{l}\text { Categorical (using the } \\
\text { median as the } \\
\text { cut-off point) }\end{array}$ & $\begin{array}{ll}- & \text { Infrequent } \\
- & \text { Frequent }\end{array}$ \\
\hline
\end{tabular}


Table 2. Cont.

\begin{tabular}{|c|c|c|c|}
\hline Theme & Variables & Type of Variable & Classification \\
\hline & $\begin{array}{l}\text { Overall score for frequency of receiving } \\
\text { health information from all sources } \\
\text { (Total score for all sources: } 1-3 \text { for each } \\
\text { question, } 8 \text { questions in total) }\end{array}$ & $\begin{array}{l}\text { Categorical (using the } \\
\text { median as the } \\
\text { cut-off point) }\end{array}$ & $\begin{array}{ll}- & \text { Infrequent } \\
- & \text { Frequent }\end{array}$ \\
\hline Knowledge & $\begin{array}{l}\text { Knowledge about disease } \\
\text { (Total score: } 0-1 \text { for each question, } \\
12 \text { questions in total) } \\
\text { (Example: Loss of smell or taste is a } \\
\text { symptom of COVID-19. (Yes/No)) }\end{array}$ & $\begin{array}{l}\text { Categorical (using the } \\
\text { median as the } \\
\text { cut-off point) }\end{array}$ & $\begin{array}{l}\text { - } \quad \text { Low level of knowledge } \\
\text { - } \quad \text { High level of knowledge }\end{array}$ \\
\hline
\end{tabular}

Awareness of public health measures (Total score: $0-1$ for each question, Awareness of public 5 questions in total) health measures (Example: If people visit Thailand from other countries, they must quarantine for at least 14 days. (Aware/Unaware))
Attitude toward public health measures

(Total score: $1-3$ for each question, 5 questions in total)

(Example: Public gatherings are prohibited during COVID-19 outbreaks. (Agree/Neutral/disagree)
Regular preventive practices for COVID-19 situation

Self-reported preventive practices
(Total score: $0-1$ of each, 7 questions in total)

(Example: I always wear a mask when I go outside. (Yes/No))
Categorical (using the median as the cut-off point)
- Low level of awareness

- High level of awareness

Perceived susceptibility

(Total score: 1-3 for each question, 3 questions in total)

(Example of questions: I consider myself to be at risk of COVID-19. (Agree/Neutral/Disagree))

Perceived severity

(Total score: 1-3 for each question, 3 questions in total) (Example: If I get COVID-19, I will

Health belief model probably die.

Categorical (using the median as the cut-off point)
- Low level of preventive practices

- High level of preventive practices
- Low level of positive attitude

- High level of positive attitude median as the cut-off point) . 


\section{Results}

\subsection{Descriptive Analysis}

A total of 303 participants were recruited, but 15 were excluded from the analysis due to failure to follow protocol such as not following the IMC question or not providing their age. Consequently, the final number of participants was 288; of those, 8 were from Phuket (3\%), 76 were from Ranong (26\%), and 204 were from Samut Sakhon (71\%). The median age of the participants was 30 ( 25 th percentile $=27,75$ th percentile $=37$ ), and the male-tofemale ratio was 1:2.1. The median length of stay in Thailand was eight years. In terms of ethnicities, most of the participants were Myanmar (63\%), followed by Mon (27\%), Dawei $(8 \%)$, and Karen (2\%). Most of them had completed primary school (58\%). Regarding occupation, factory workers (49\%) constituted the majority of the participants. Most of the participants $(65 \%)$ had income levels lower than the provincial minimum wage. In terms of Thai comprehension, $55 \%$ of the participants were partially fluent in speaking Thai, while $62 \%$ of the participants could not read Thai. For insurance, the majority of participants $(64 \%)$ were insured by the Social Security Scheme (the main public insurance scheme for formal-sector workers). About one-fifth (22\%) were uninsured, and approximately $13 \%$ were insured by the Health Insurance Card Scheme (the main public insurance scheme for informal-sector migrants, managed by the Ministry of Public Health).

A large proportion of participants exhibited a high level of access to resources supporting exposure to health information. Approximately $99 \%$ of participants had access to electricity, $94 \%$ had phone signals, and $92 \%$ had internet connectivity. Access to resources supporting preventive practices was also high, with $99 \%$ of the participants having access to face masks and about $88 \%$ having access to alcohol-based sanitizers; access to soap and tap water was also high, at approximately $97 \%$ and $94 \%$, respectively. In terms of information dissemination, social media was the most common source of health information (67\%); the second and third most common sources were from MHW/MHV/VHV (63\%) and health professionals (56\%), respectively (see Figure 1 ).

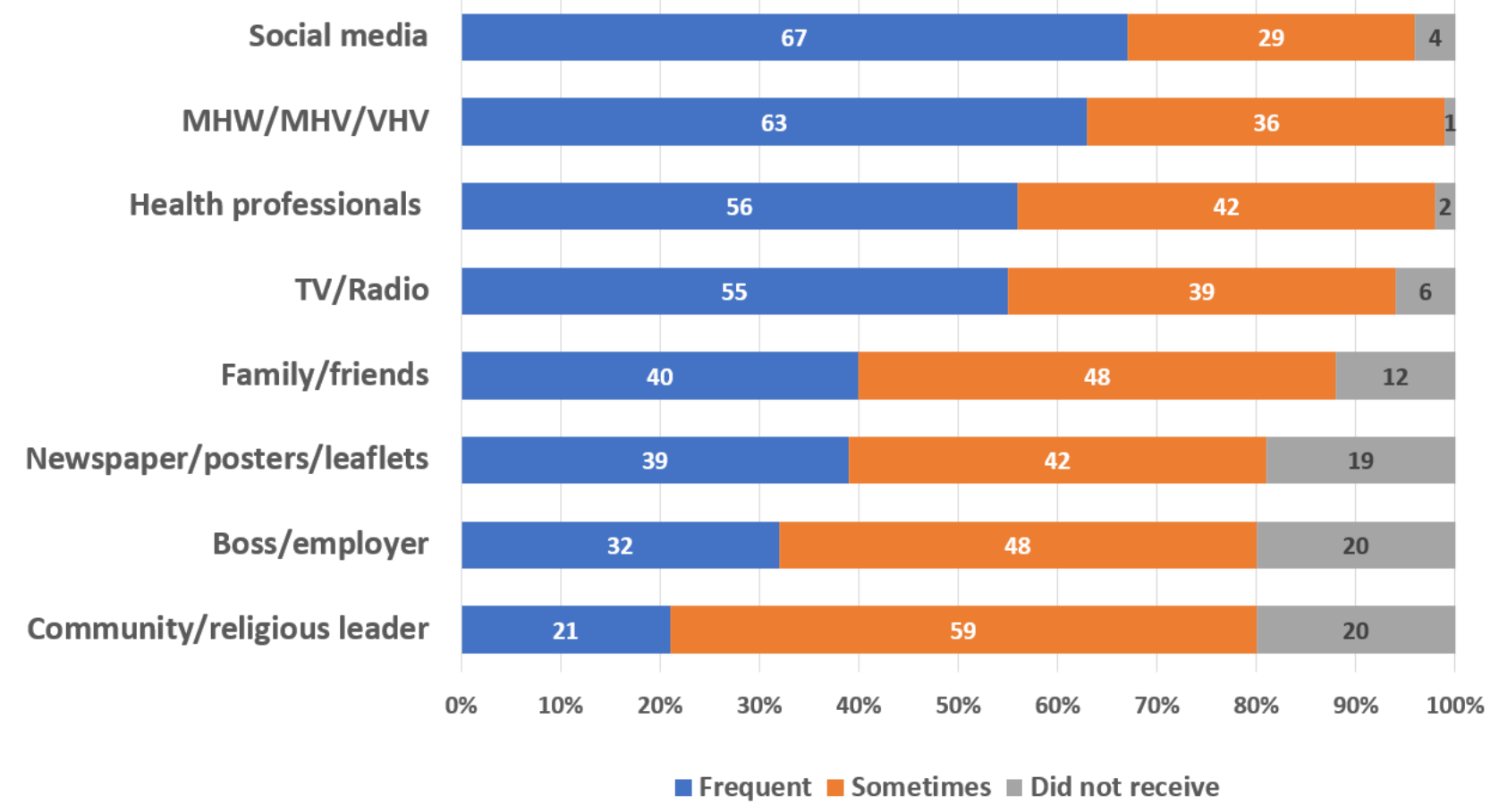

Figure 1. Proportion and frequency of health information received based on communication channels: MHW, migrant health worker; MHV, migrant health volunteer; VHV, village health volunteer. 
The majority of participants reported a high score on many questions such as awareness of public health measures (85\%) and preventive practices (77\%), followed by knowledge $(68 \%)$, attitude $(63 \%)$, and health literacy $(58 \%)$. The percentage of participants that reported receiving health information frequently from all sources combined was $54 \%$. For the health belief model constructs, most participants had low scores for perceived susceptibility $(75 \%)$, perceived barriers $(71 \%)$, and perceived severity $(66 \%)$. More details are shown in Figure 2.

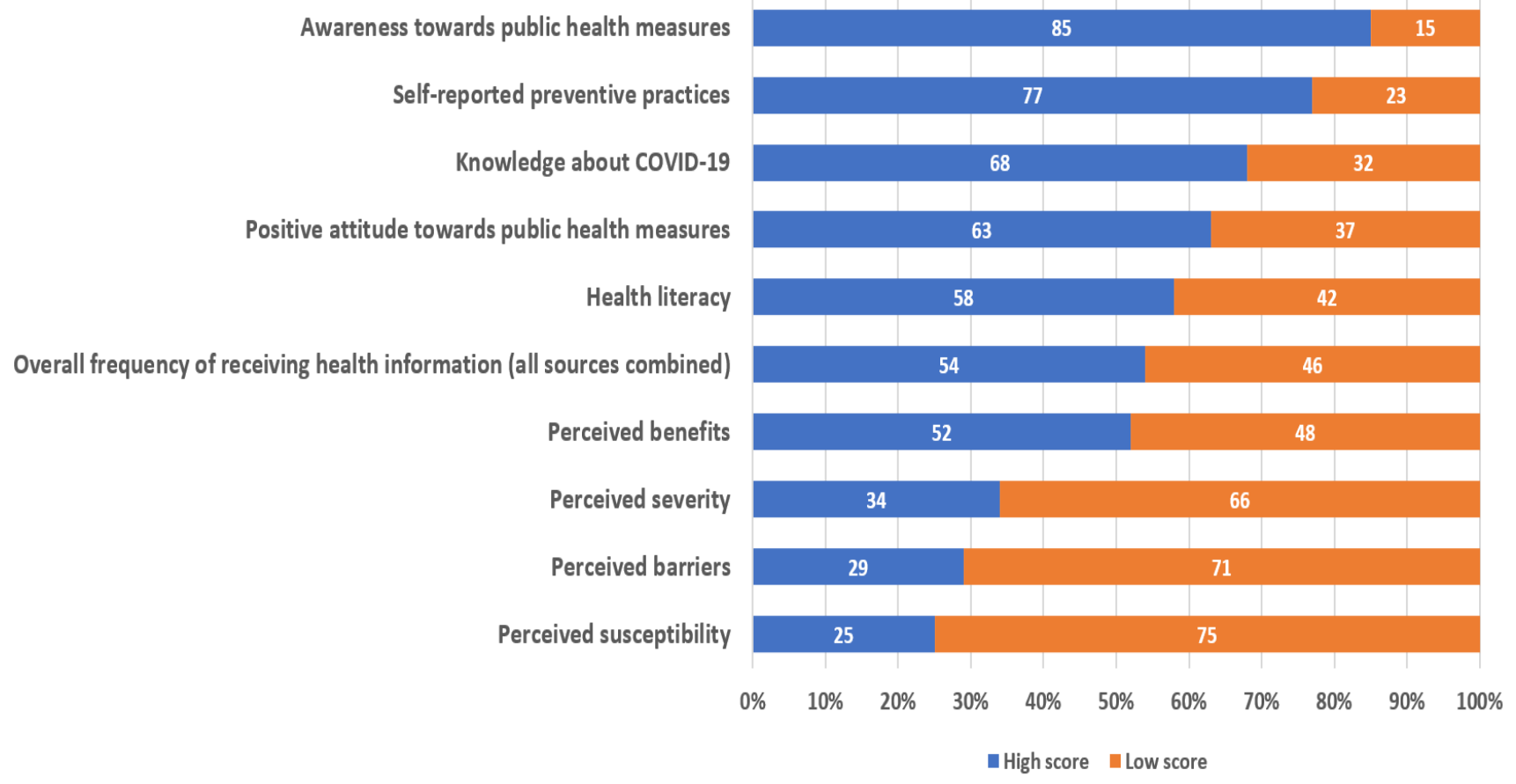

Figure 2. Proportion of scores for the variables.

\subsection{Inferential Analysis}

Univariate analysis found a significant variation in the awareness of public health measures based on the length of stay in the country ( $p$-value $=0.03$ ). Other independent variables for awareness of public health measures did not show statistically significant associations (see Table 3).

Table 3. Univariate analysis on awareness of public health measures on COVID-19.

\begin{tabular}{lccc}
\hline Independent Factors & $\begin{array}{c}\text { High Level of Awareness, } \\
\mathbf{n}(\%)\end{array}$ & $\begin{array}{c}\text { Low Level of Awareness, } \\
\mathbf{n}(\%)\end{array}$ & $p$-Value \\
\hline Gender & & & \\
$\quad$ Female & $157(83)$ & $33(17)$ & 0.59 \\
$\quad$ Male & $83(89)$ & $10(11)$ & \\
\hline Age (years) & & & \\
$\quad<25$ & $31(82)$ & $7(18)$ & 0.42 \\
$25-59$ & $207(85)$ & $36(15)$ & \\
$\geq 60$ & $3(100)$ & 0 & 0.72 \\
\hline Ethnicity & $155(87)$ & $24(13)$ & \\
$\quad$ Myanmar & $84(82)$ & $19(18)$ & 0.59 \\
$\quad$ Non-Myanmar & $4(80)$ & $1(20)$ & \\
\hline Province & $68(91)$ & $7(9)$ & \\
$\quad$ Phuket & $169(83)$ & $35(17)$ & \\
$\quad$ Ranong & & & \\
$\quad$ Samut Sakhon &
\end{tabular}


Table 3. Cont.

\begin{tabular}{|c|c|c|c|}
\hline Independent Factors & $\begin{array}{l}\text { High Level of Awareness, } \\
\text { n (\%) }\end{array}$ & $\begin{array}{c}\text { Low Level of Awareness, } \\
\text { n (\%) }\end{array}$ & $p$-Value \\
\hline \multicolumn{4}{|l|}{ Length of stay in Thailand (years) } \\
\hline$\leq 8$ & $135(91)$ & $13(9)$ & \multirow{2}{*}{0.03} \\
\hline$>8$ & $103(77)$ & $30(23)$ & \\
\hline \multicolumn{4}{|l|}{ Education } \\
\hline No formal education & $29(88)$ & $4(12)$ & \multirow{3}{*}{0.44} \\
\hline Primary school & $135(82)$ & $29(18)$ & \\
\hline Secondary school and upper & $77(89)$ & $10(11)$ & \\
\hline \multicolumn{4}{|l|}{ Occupation } \\
\hline Unemployed & $41(77)$ & $12(23)$ & \multirow{5}{*}{0.27} \\
\hline Factory worker & $119(84)$ & $22(16)$ & \\
\hline Agriculture/fishery & $25(93)$ & $2(7)$ & \\
\hline Construction worker & $38(95)$ & $2(5)$ & \\
\hline Business/housemaid/waiter & $18(78)$ & $5(22)$ & \\
\hline \multicolumn{4}{|l|}{ Thai Reading Comprehension } \\
\hline Cannot understand & $143(81)$ & $33(19)$ & \multirow{3}{*}{0.15} \\
\hline Partially understand & $84(89)$ & $10(11)$ & \\
\hline Fully understand & $13(100)$ & 0 & \\
\hline \multicolumn{4}{|l|}{ Thai Listening Comprehension } \\
\hline Cannot understand & $46(90)$ & $5(10)$ & \multirow{3}{*}{0.41} \\
\hline Partially understand & $134(87)$ & $20(13)$ & \\
\hline Fully understand & $60(77)$ & $18(23)$ & \\
\hline \multicolumn{4}{|l|}{ Income } \\
\hline Lower than minimum wage & $153(83)$ & $32(17)$ & \multirow{3}{*}{0.26} \\
\hline Equal to minimum wage & $13(100)$ & 0 & \\
\hline Higher than minimum wage & $75(87)$ & $11(13)$ & \\
\hline \multicolumn{4}{|l|}{ Health insurance } \\
\hline No insurance/unknown & $48(76)$ & $15(24)$ & \multirow{3}{*}{0.10} \\
\hline Health Insurance Card Scheme & $30(83)$ & $6(17)$ & \\
\hline Social Security Scheme & $163(88)$ & $22(12)$ & \\
\hline \multicolumn{4}{|c|}{ Resources supporting access to information } \\
\hline Low access & $23(85)$ & $4(15)$ & \multirow[t]{2}{*}{0.96} \\
\hline High access & $217(85)$ & $38(15)$ & \\
\hline \multicolumn{4}{|l|}{ Family member (aged $\geq 15$ years) } \\
\hline$<2$ people & $14(100)$ & 0 & \multirow[t]{2}{*}{$\mathrm{N} / \mathrm{A}$} \\
\hline$\geq 2$ people & $168(82)$ & $38(18)$ & \\
\hline \multicolumn{4}{|c|}{ Receiving health information from health professionals } \\
\hline Infrequent & $117(87)$ & $18(13)$ & \multirow[t]{2}{*}{0.37} \\
\hline Frequent & $124(83)$ & $25(17)$ & \\
\hline \multicolumn{4}{|c|}{ Receiving health information from community members } \\
\hline Infrequent & $54(84)$ & $10(16)$ & \multirow[t]{2}{*}{0.62} \\
\hline Frequent & $186(85)$ & $33(15)$ & \\
\hline \multicolumn{4}{|c|}{ Receiving health information from public mass media } \\
\hline Infrequent & $20(80)$ & $5(20)$ & 0.75 \\
\hline Frequent & $220(86)$ & $37(14)$ & \\
\hline Receiving health information from soc & & & \\
\hline Infrequent & $76(80)$ & $19(20)$ & 0.42 \\
\hline Frequent & $165(87)$ & $24(13)$ & \\
\hline
\end{tabular}

For preventive practices, gender $(p=0.04)$, age groups $(p=0.03)$, education $(p=0.05)$, occupation $(p=0.03)$, Thai reading comprehension $(p<0.01)$, health insurance $(p<0.01)$, frequency of receiving health information $(p<0.01)$, and perceived barriers $(p=0.03)$ showed statistically significant associations. More details are described in Table 4 . 
Table 4. Univariate analysis on self-reported preventive practices during COVID-19.

\begin{tabular}{|c|c|c|c|}
\hline Independent Factors & $\begin{array}{c}\text { High Level of } \\
\text { Preventive Practices, } \mathrm{n}(\%)\end{array}$ & $\begin{array}{c}\text { Low Level of } \\
\text { Preventive Practices, } \\
\text { n (\%) }\end{array}$ & $p$-Value \\
\hline \multicolumn{4}{|l|}{ Gender } \\
\hline Female & $140(73)$ & $51(27)$ & \multirow[t]{2}{*}{0.04} \\
\hline Male & $79(85)$ & $14(15)$ & \\
\hline \multicolumn{4}{|l|}{ Age (years) } \\
\hline$<25$ & $35(95)$ & $2(5)$ & \multirow{3}{*}{0.03} \\
\hline $25-59$ & $182(74)$ & $63(26)$ & \\
\hline$\geq 60$ & $3(100)$ & 0 & \\
\hline \multicolumn{4}{|l|}{ Ethnicity } \\
\hline Myanmar & $145(81)$ & $34(19)$ & \multirow[t]{2}{*}{0.09} \\
\hline Non-Myanmar & $74(70)$ & $31(30)$ & \\
\hline \multicolumn{4}{|l|}{ Province } \\
\hline Phuket & $8(100)$ & 0 & \multirow{3}{*}{0.45} \\
\hline Ranong & $56(77)$ & $17(23)$ & \\
\hline Samut Sakhon & $156(76)$ & $48(24)$ & \\
\hline \multicolumn{4}{|l|}{ Length of stay in Thailand (years) } \\
\hline$\leq 8$ & $117(78)$ & $33(22)$ & \multirow[t]{2}{*}{0.90} \\
\hline$>8$ & $101(77)$ & $31(23)$ & \\
\hline \multicolumn{4}{|l|}{ Education } \\
\hline No formal education & $21(64)$ & $12(36)$ & \multirow{3}{*}{0.05} \\
\hline Primary school & $128(77)$ & $38(23)$ & \\
\hline Secondary school and upper & $71(83)$ & $15(17)$ & \\
\hline \multicolumn{4}{|l|}{ Occupation } \\
\hline Unemployed & $34(65)$ & $18(35)$ & \multirow{5}{*}{0.03} \\
\hline Factory worker & $111(79)$ & $30(21)$ & \\
\hline Agriculture/fishery & $18(64)$ & $10(36)$ & \\
\hline Construction worker & $38(93)$ & $3(7)$ & \\
\hline Business/housemaid/waiter & $19(83)$ & $4(17)$ & \\
\hline \multicolumn{4}{|l|}{ Thai Reading Comprehension } \\
\hline Cannot understand & $126(71)$ & $51(29)$ & \multirow{3}{*}{$<0.01$} \\
\hline Partially understand & $80(85)$ & $14(15)$ & \\
\hline Fully understand & $13(100)$ & 0 & \\
\hline \multicolumn{4}{|l|}{ Thai Listening Comprehension } \\
\hline Cannot understand & $38(73)$ & $14(27)$ & \multirow{3}{*}{0.65} \\
\hline Partially understand & $123(80)$ & $31(20)$ & \\
\hline Fully understand & $58(74)$ & $20(26)$ & \\
\hline \multicolumn{4}{|l|}{ Income } \\
\hline Lower than minimum wage & $142(76)$ & $44(24)$ & \multirow{3}{*}{0.16} \\
\hline Equal to minimum wage & $5(38)$ & $8(62)$ & \\
\hline Higher than minimum wage & $73(85)$ & $13(15)$ & \\
\hline \multicolumn{4}{|l|}{ Health insurance } \\
\hline No insurance/unknown & $39(62)$ & $24(38)$ & \multirow{3}{*}{$<0.01$} \\
\hline Health Insurance Card Scheme & $30(83)$ & $6(17)$ & \\
\hline Social Security Scheme & $151(81)$ & $35(19)$ & \\
\hline Resources supporting access to preven & & & \\
\hline Low access & $37(77)$ & $11(23)$ & 0.39 \\
\hline High access & $181(77)$ & $54(23)$ & \\
\hline Family member (age $\geq 15$ years old) & & & \\
\hline$<2$ people & $12(86)$ & $2(14)$ & 0.31 \\
\hline$\geq 2$ people & $149(72)$ & $58(28)$ & \\
\hline
\end{tabular}


Table 4. Cont.

\begin{tabular}{|c|c|c|c|}
\hline Independent Factors & $\begin{array}{c}\text { High Level of } \\
\text { Preventive Practices, n (\%) }\end{array}$ & $\begin{array}{c}\text { Low Level of } \\
\text { Preventive Practices, } \\
\text { n (\%) }\end{array}$ & $p$-Value \\
\hline $\begin{array}{l}\text { Overall frequency of receiving health information } \\
\text { (all sources combined) } \\
\text { Infrequent } \\
\text { Frequent }\end{array}$ & $\begin{array}{c}88(68) \\
129(85)\end{array}$ & $\begin{array}{l}42(32) \\
22(15)\end{array}$ & $<0.01$ \\
\hline $\begin{array}{l}\text { Health literacy } \\
\text { Low health literacy } \\
\text { High health literacy }\end{array}$ & $\begin{array}{c}91(77) \\
127(77)\end{array}$ & $\begin{array}{l}27(23) \\
37(23)\end{array}$ & 0.73 \\
\hline $\begin{array}{l}\text { Positive attitude toward public health measures } \\
\text { Low level of positive attitude } \\
\text { High level of positive attitude }\end{array}$ & $\begin{array}{c}80(78) \\
136(77)\end{array}$ & $\begin{array}{l}23(22) \\
41(23)\end{array}$ & 0.69 \\
\hline $\begin{array}{l}\text { Knowledge about disease } \\
\text { Low level of knowledge } \\
\text { High level of knowledge }\end{array}$ & $\begin{array}{c}65(72) \\
155(79)\end{array}$ & $\begin{array}{l}25(28) \\
40(21)\end{array}$ & 0.33 \\
\hline $\begin{array}{l}\text { Awareness of public health measures } \\
\text { Low level of awareness } \\
\text { High level of awareness }\end{array}$ & $\begin{array}{c}28(65) \\
188(79)\end{array}$ & $\begin{array}{l}15(35) \\
50(21)\end{array}$ & 0.33 \\
\hline $\begin{array}{l}\text { Perceived susceptibility } \\
\text { Low / moderate perception } \\
\text { High perception }\end{array}$ & $\begin{array}{l}160(75) \\
60(83)\end{array}$ & $\begin{array}{l}52(25) \\
12(17)\end{array}$ & 0.08 \\
\hline $\begin{array}{l}\text { Perceived severity } \\
\text { Low/moderate perception } \\
\text { High perception }\end{array}$ & $\begin{array}{l}140(74) \\
79(83)\end{array}$ & $\begin{array}{l}49(26) \\
16(17)\end{array}$ & 0.10 \\
\hline $\begin{array}{l}\text { Perceived benefits } \\
\text { Low/moderate perception } \\
\text { High perception }\end{array}$ & $\begin{array}{l}102(76) \\
117(79)\end{array}$ & $\begin{array}{l}33(24) \\
32(21)\end{array}$ & 0.83 \\
\hline $\begin{array}{l}\text { Perceived barriers } \\
\text { Low / moderate perception } \\
\text { High perception }\end{array}$ & $\begin{array}{c}149(74) \\
70(85)\end{array}$ & $\begin{array}{l}53(26) \\
12(15)\end{array}$ & 0.03 \\
\hline
\end{tabular}

We included the length of stay in Thailand and health insurance status in the multivariate analysis, as both variables had a $p$-value of less than or equal to 0.10 in the univariate analysis. The multivariate analysis revealed that a long length of stay in Thailand (more than 8 years (median)) was significantly associated with low awareness of public health measures to tackle the COVID-19 situation (AOR $=0.43,95 \%$ CI 0.19-0.95) (Table 5).

Table 5. Multivariate analysis on the awareness of public health measures during COVID-19.

\begin{tabular}{lcc}
\hline \multicolumn{1}{c}{ Selected Factors } & Adjusted OR (95\% CI) & $p$-Value \\
\hline $\begin{array}{l}\text { Length of stay in Thailand } \\
\leq 8 \text { years }\end{array}$ & reference & \\
$>8$ years & $0.43(0.19-0.95)$ & 0.04 \\
\hline Insurance & reference & \\
$\quad$ No insurance/unknown & $2.02(0.81-5.06)$ & reference \\
Social Security scheme & $1.78(0.54-5.85)$ & 0.13 \\
Health insurance card scheme & 0.35 \\
\hline
\end{tabular}

For the effects on preventive practices, being between 25 and 59 years of age was associated with low preventive practices (compared with being less than 25 years of age) ( $\mathrm{AOR}=0.15,95 \% \mathrm{CI} 0.03-0.72$ ). In contrast, achieving primary education (compared with a migrant worker with no formal education) $(\mathrm{AOR}=3.24,95 \% \mathrm{CI} 1.18-8.93)$, and frequent 
acquisition of health information from all sources (AOR $=4.20,95 \%$ CI $1.95-9.03$ ) were significantly associated with high levels of preventive practices (Table 6).

Table 6. Multivariate analysis on self-reported preventive practices during COVID-19.

\begin{tabular}{|c|c|c|}
\hline Selected Factors & Adjusted OR (95\% CI) & $p$-Value \\
\hline \multicolumn{3}{|l|}{ Gender } \\
\hline Female & reference & \\
\hline Male & $1.08(0.46-2.54)$ & 0.86 \\
\hline \multicolumn{3}{|l|}{ Age (years) } \\
\hline$<25$ & reference & \\
\hline $25-59$ & $0.15(0.03-0.72)$ & 0.02 \\
\hline$\geq 60$ & NA & NA \\
\hline \multicolumn{3}{|l|}{ Ethnicity } \\
\hline Myanmar & reference & \\
\hline Non-Myanmar & $0.63(0.29-1.35)$ & 0.24 \\
\hline \multicolumn{3}{|l|}{ Education } \\
\hline No formal education & reference & \\
\hline Primary school & $3.24(1.18-8.93)$ & 0.02 \\
\hline Secondary school and upper & $2.64(0.85-8.16)$ & 0.09 \\
\hline \multicolumn{3}{|l|}{ Occupation } \\
\hline Unemployed & reference & \\
\hline Factory worker & $0.46(0.09-2.49)$ & 0.37 \\
\hline Agriculture/fishery & $0.38(0.09-1.59)$ & 0.18 \\
\hline Construction worker & $2.20(0.31-15.51)$ & 0.43 \\
\hline Business/housemaid/waiter & $0.77(0.16-3.59)$ & 0.74 \\
\hline \multicolumn{3}{|l|}{ Thai Reading Comprehension } \\
\hline Cannot understand & reference & \\
\hline Partially understand & $1.71(0.72-4.05)$ & 0.22 \\
\hline Fully understand & NA & NA \\
\hline \multicolumn{3}{|l|}{ Insurance } \\
\hline No insurance/unknown & reference & \\
\hline Health insurance card scheme & $2.46(0.64-9.43)$ & 0.19 \\
\hline Social Security scheme & $2.49(0.58-10.62)$ & 0.22 \\
\hline \multicolumn{3}{|l|}{$\begin{array}{l}\text { Overall frequency of receiving health } \\
\text { information (all sources combined) }\end{array}$} \\
\hline Infrequent & reference & \\
\hline Frequent & $4.20(1.95-9.03)$ & $<0.01$ \\
\hline \multicolumn{3}{|l|}{ Perceived susceptibility } \\
\hline Low/moderate perception & reference & \\
\hline High perception & $0.98(0.37-2.60)$ & 0.97 \\
\hline \multicolumn{3}{|l|}{ Perceived severity } \\
\hline Low/moderate perception & reference & \\
\hline High perception & $1.18(0.47-3.00)$ & 0.72 \\
\hline \multicolumn{3}{|l|}{ Perceived barriers } \\
\hline Low/moderate perception & reference & \\
\hline High perception & $0.97(0.38-2.48)$ & 0.95 \\
\hline
\end{tabular}

Note: NA = dropped from the analysis due to perfect prediction.

\section{Discussion}

Overall, we found that participants had high levels of awareness toward public health measures and preventive practices. The most common source of health information was social media. Based on the multivariate analysis, we found that a longer stay in Thailand had a significant association with lower awareness of public health measures. Frequent reception of health information and primary school completion showed a statistically significant association with a high level of preventive practices, relative to those who rarely 
received health information and had no formal schooling. However, being middle-aged (compared with being young) was significantly associated with fewer preventive practices.

Our study also found that a longer stay in Thailand was associated with lower awareness about public health measures. One possible explanation might be that migrants who have lived in the host country for a longer period were more familiar with the lifestyle and may be less sensitive to new information. In addition, a longer stay was associated with multiple morbidities. To date, there is no clear explanation about this phenomenon but some studies in the literature have ascribed this to chronic stress, discrimination, or other factors associated with post-migration experiences [31,32].

A high frequency of receiving health information likely amplified the perception of risk and led to higher levels of preventive practices. Frequent communication via various channels had both direct and indirect effects on preventive behaviors, especially during the pandemic [33]. Therefore, the government should not overly rely on the delivery of health messages through a single particular channel but should distribute the information through multiple channels to ensure migrants engage in desired preventive behaviors.

From the findings above, middle-aged participants were likely to have lower levels of preventive practices compared with younger participants. According to the association between longer stay and low level of public health measure awareness, it could be inferred that as older migrants were more likely to have longer stay in the host country, they probably ignored or did not follow the public health measures. Evidence from other countries showed that migrants with a longer stay or those of older age tended to face greater health risks, compared with younger migrants [34]. Migrant workers with primary education were likely to exhibit higher degrees of preventive practices, compared with those with no formal education; having no formal education leads to low comprehension or misunderstandings about health messages, which may ultimately result in low levels of preventive practices [35]. Therefore, risk communication should not overlook migrants who have difficulties in accessing information due to low literacy, as well as those who are most likely to ignore or not conform to public health measures such as older migrants and migrants who have stayed in Thailand for more than 8 years.

This study was among the first studies to explore the importance of health risk communication for migrants during the COVID-19 pandemic. The use of empirical data was one of the main strengths of the study. However, it also contained certain limitations. First, we chose to use the median as a cut-off value to transform a multilevel categorical variable into a binary variable. Although this approach was able to facilitate the interpretation of results to a wide range of audiences, it might have also caused residual confounding. This issue also flags the need for further research, as there are no standard questionnaires to assess the level of health information reception among migrants thus far. Second, generalization of the findings is quite limited as the migrants in this study were mostly from Myanmar, while migrants in other settings (for example, those in the northeast or north of Thailand) are likely to have their own demographic characteristics and behaviors. Third, we could not guarantee our samples were free from selection bias. Despite our intent to ask local coordinators to randomly select migrants in the community to take part in the study, it is possible that the coordinators may have selected participants who were closer to them.

\section{Conclusions}

Among migrant workers in Thailand, the frequent reception of health information through various channels is positively linked with better preventive practices against COVID-19. Further initiatives to promote the education and health literacy of migrants should focus more on migrants with no formal education and migrants who are likely to ignore or not comply with public health measures. The government should deliver health messages through various channels to encourage migrants to engage in desired preventive behaviors. Further qualitative studies may overcome the limitations of this study. 
Author Contributions: Conceptualization, R.P., H.K. and R.S.; methodology, R.S., R.P. and H.K.; software, R.P., H.K. and R.S.; validation, R.P., H.K. and R.S.; formal analysis, R.P., H.K. and R.S.; investigation, H.K., R.P., P.S., M.P. and S.J.; resources, H.K. and P.S.; data curation, R.P. and H.K.; writing—original draft preparation, R.P. and M.P.; writing—review and editing, R.P., H.K., P.S., M.P., S.J. and R.S.; visualization, R.P.; supervision, R.S.; project administration, R.P. and H.K.; funding acquisition, H.K. and P.S. All authors have read and agreed to the published version of the manuscript.

Funding: This research was funded by Health Systems Research Institute (Grant Number 63-162).

Institutional Review Board Statement: This study received ethics approval from the Institute for the Development of Human Research Protections, Thailand (IHRP 985/2563). Anonymity and confidentially were strictly maintained. The data collection and data analysis were conducted according to the principles expressed in the Declaration of Helsinki.

Informed Consent Statement: Informed consent was obtained before the process of the study.

Data Availability Statement: The datasets used in this article are not available publicly; however, they may be available upon reasonably request to IHPP.

Acknowledgments: The authors would like to express their appreciation to The Provincial Health Office of Phuket, Ranong, and Samut Sakhon, as well as the Raks Thai Foundation and World Vision Foundation of Thailand, for their considerable support and good cooperation.

Conflicts of Interest: The authors declare no conflict of interest.

\section{References}

1. Huang, C.; Wang, Y.; Li, X.; Ren, L.; Zhao, J.; Hu, Y.; Zhang, L.; Fan, G.; Xu, J.; Gu, X.; et al. Clinical features of patients infected with 2019 novel coronavirus in Wuhan, China. Lancet 2020, 395, 497-506. [CrossRef]

2. Jee, Y. WHO International Health Regulations Emergency Committee for the COVID-19 outbreak. Epidemiol. Health 2020, 42, e2020013. [CrossRef] [PubMed]

3. World Health Organization. Listings of WHO's Response to COVID-19. Available online: https://www.who.int/news/item/29 -06-2020-covid timeline (accessed on 15 February 2021).

4. World Health Organization. Statement on the Second Meeting of the International Health Regulations (2005) Emergency Committee Regarding the Outbreak of Novel Coronavirus (2019-nCoV). Available online: https://www.who.int/news-room/ detail/30-01-2020-statement-on-the-second-meeting-of-the-international-health-regulations-(2005)-emergency-committeeregarding-the-outbreak-of-novel-coronavirus-(2019-ncov) (accessed on 19 May 2021).

5. World Health Organization. WHO Coronavirus (COVID-19) Dashboard. Available online: https://covid19.who.int/ (accessed on 21 October 2021).

6. $\quad$ Doung-Ngern, P.; Suphanchaimat, R.; Panjangampatthana, A.; Janekrongtham, C.; Ruampoom, D.; Daochaeng, N.; Eungkanit, N.; Pisitpayat, N.; Srisong, N.; Yasopa, O.; et al. Case-Control Study of Use of Personal Protective Measures and Risk for SARS-CoV 2 Infection, Thailand. Emerg. Infect. Dis. 2020, 26, 2607-2616. [CrossRef] [PubMed]

7. Namwat, C.; Suphanchaimat, R.; Nittayasoot, N.; Iamsirithaworn, S. Thailand's Response against Coronavirus Disease 2019: Challenges and Lessons Learned. OSIR 2020, 13, 33-37.

8. World Health Organization. Risk Communication. Available online: https://www.who.int/risk-communication/PIP_brochure_ EN_lo.pdf (accessed on 30 May 2021).

9. Ministry of Public Health. COVID-19 Infodemic Management: Thailand Experience. The 75th Session of the General Assembly of the United Nations, 23 September 2020; WHO: Geneva, Switzerland, 2020. Available online: https:/ /www.who.int/docs/default-source/ coronaviruse /risk-comms-updates / thailand-unga-presentation-infodemic-thailand-21sep2020-final.pdf?sfvrsn=d757509e_6 (accessed on 30 May 2021).

10. Tabari, P.; Amini, M.; Moghadami, M.; Moosavi, M. International Public Health Responses to COVID-19 Outbreak: A Rapid Review. Iran. J. Med. Sci. 2020, 45, 157-169. [PubMed]

11. Marome, W.; Shaw, R. COVID-19 Response in Thailand and Its Implications on Future Preparedness. Int. J. Environ. Res. Public Health 2021, 18, 1089. [CrossRef] [PubMed]

12. WHO Thailand Country Office Coronavirus Disease 2019: Situation Report. Available online: https://cdn.who.int/media/docs/ default-source/searo/thailand/2021_1_25_eng_sitrep_135-covid19.pdf?sfvrsn=a034c3d5_3 (accessed on 15 February 2021).

13. Luksamijarulkul, P.; Suknongbung, S.; Vatanasomboon, P.; Sujirarut, D. Health status, environmental living conditions and microbial indoor air quality among migrant worker households in Thailand. Southeast Asian J. Trop. Med. Public Health 2017, 48, 396-406. [PubMed]

14. United Nations. Thematic Working Group on Migration in Thailand. Thailand Migration Report 2019; Bangkok, Thailand, 2019. Available online: https://thailand.iom.int/sites/thailand/files/document/publications/Thailand\%20Report\%202019_22012 019_HiRes.pdf (access on 14 April 2021). 
15. Office of Foreign Workers Administration. Statistics of Remaining Cross-Border Migrants Holding Work Permit in Thailand as of September 2021; Department of Employment, Ministry of Labor: Bangkok, Thailand, 2021. Available online: https://www.doe.go. th/prd/assets/upload/files/alien_th/e7ecaa17a00907e4eefe2a5a81462935.pdf (accessed on 21 October 2021).

16. Koh, D. Migrant workers and COVID-19. Occup. Environ. Med. 2020, 77, 634-636. [CrossRef] [PubMed]

17. World Health Organization. Interim Guidance for Refugee and Migrant Health in Relation to COVID-19 in the WHO European Region. Available online: https:/ / www.euro.who.int/_data/assets/pdf_file/0008/434978/Interim-guidance-refugee-andmigrant-health-COVID-19.pdf (accessed on 17 June 2021).

18. Hickey, J.; Gagnon, A.J.; Jitthai, N. Pandemic preparedness: Perceptions of vulnerable migrants in Thailand towards WHOrecommended non-pharmaceutical interventions: A cross-sectional study. BMC Public Health 2014, 14, 665. [CrossRef] [PubMed]

19. Hickey, J.E.; Gagnon, A.J.; Jitthai, N. Knowledge about pandemic influenza preparedness among vulnerable migrants in Thailand. Health Promot. Int. 2016, 31, 124-132. [CrossRef] [PubMed]

20. International Organization for Migration, Thailand. Rapid Assessment: COVID-19-Related Vulnerabilities and Perceptions of Non-Thai Populations in Thailand; Bangkok, April 2020. Available online: https://thailand.iom.int/rapid-assessment-covid-19 -related-vulnerabilities-and-perceptions-non-thai-population-thailand (accessed on 10 April 2021).

21. International Organization for Migration, Thailand. Rapid Assessment: COVID-19 Related Vulnerabilities and Perceptions of Non-Thai Populations in Thailand (round 2); Bangkok, June 2020. Available online: https://reliefweb.int/report/thailand/rapidassessment-round-2-covid-19-related-vulnerabilities-and-perceptions-non-thai (accessed on 16 April 2021).

22. International Organization for Migration, Thailand. Rapid Assessment: COVID-19 Related Vulnerabilities and Perceptions of Non-Thai Populations in Thailand (round 3); Bangkok, November 2020. Available online: https://thailand.iom.int/rapidassessment-round-3-covid-19-related-vulnerabilities-and-perceptions-non-thai-populations (accessed on 16 April 2021).

23. Jones, C.L.; Jensen, J.D.; Scherr, C.L.; Brown, N.R.; Christy, K.; Weaver, J. The Health Belief Model as an explanatory framework in communication research: Exploring parallel, serial, and moderated mediation. Health Commun. 2015, 30, 566-576. [CrossRef] [PubMed]

24. Bauza, V.; Sclar, G.D.; Bisoyi, A.; Owens, A.; Ghugey, A.; Clasen, T. Experience of the COVID-19 Pandemic in Rural Odisha, India: Knowledge, Preventative Actions, and Impacts on Daily Life. Int. J. Environ. Res. Public Health 2021, 18, 2863. [CrossRef] [PubMed]

25. Department of Disease Control. Corona Virus Disease (COVID-19). Available online: https://ddc.moph.go.th/viralpneumonia/ eng/index.php (accessed on 1 March 2021).

26. Thai Government. Centre for COVID-19 Situation Administration. Available online: https://www.moicovid.com/ (accessed on 1 March 2021).

27. Osborne, R.H.; Batterham, R.W.; Elsworth, G.R.; Hawkins, M.; Buchbinder, R. The grounded psychometric development and initial validation of the Health Literacy Questionnaire (HLQ). BMC Public Health 2013, 13, 658. [CrossRef] [PubMed]

28. Ministry of Labor. Minimum Wage. Available online: https://www.mol.go.th/en/minimum-wage (accessed on 1 April 2021).

29. Simpson, R.M.; Knowles, E.; O'Cathain, A. Health literacy levels of British adults: A cross-sectional survey using two domains of the Health Literacy Questionnaire (HLQ). BMC Public Health 2020, 20, 1819. [CrossRef] [PubMed]

30. Didarloo, A.; Nabilou, B.; Khalkhali, H.R. Psychosocial predictors of breast self-examination behavior among female students: An application of the health belief model using logistic regression. BMC Public Health 2017, 17, 861. [CrossRef]

31. Doamekpor, L.A.; Dinwiddie, G.Y. Allostatic load in foreign-born and US-born blacks: Evidence from the 2001-2010 National Health and Nutrition Examination Survey. Am. J. Public Health 2015, 105, 591-597. [CrossRef]

32. McEwen, B.S. Protection and damage from acute and chronic stress: Allostasis and allostatic overload and relevance to the pathophysiology of psychiatric disorders. Ann. N. Y. Acad. Sci. 2004, 1032, 1-7. [CrossRef] [PubMed]

33. Heydari, S.T.; Zarei, L.; Sadati, A.K.; Moradi, N.; Akbari, M.; Mehralian, G.; Lankarani, K.B. The effect of risk communication on preventive and protective Behaviours during the COVID-19 outbreak: Mediating role of risk perception. BMC Public Health 2021, 21, 54. [CrossRef] [PubMed]

34. Lee, C.H.; Yun, J.M.; Han, J.S.; Park, S.M.; Park, Y.S.; Hong, S.K. The Prevalence of Chronic Diseases among Migrants in Korea According to Their Length of Stay and Residential Status. Korean J. Fam. Med. 2012, 33, 34-43. [CrossRef] [PubMed]

35. Gilder, M.E.; Moo, P.; Hashmi, A.; Praisaengdet, N.; Wai, K.; Pimanpanarak, M.; Carrara, V.I.; Angkurawaranon, C.; Jiraporncharoen, W.; McGready, R. "I can't read and don't understand": Health literacy and health messaging about folic acid for neural tube defect prevention in a migrant population on the Myanmar-Thailand border. PLoS ONE 2019, 14, e0218138. [CrossRef] 